\title{
THE DEVELOPMENT OF THE THEORY OF DEFICIENT VALUES SINCE NEVANLINNA
}

\author{
W. H. J. FUCHS
}

1. It is the hallmark of a very great mathematician that he creates concepts which throw new light on old problems and which give direction to later work. In this talk I want to pay homage to R. Nevanlinna by tracing the role which his concept of "deficient value" has played in the recent theory of meromorphic function.

The literature on this subject is enormous. Limitations of time, space and my own competence force me to concentrate strictly on theorems concerned with the "fine structure" of meromorphic functions which is revealed by the notion of deficiency. I have to omit many relevant topics such as Valiron deficiencies or theorems in the theory of differential equations. I shall completely ignore fields outside classical function theory in whose development the notion of deficiency has played a crucial part: af Hällström's Nevanlinna theory for multiply connected regions, mapping of Riemann surfaces (Sario), mapping of complex manifolds into complex manifolds (Bott, Chern, Griffiths, Stoll). I shall even be silent about the theory of holomorphic curves created by L. Ahlfors although there are several recent papers which develop striking analogies of this theory and the classical theory of deficiencies. The bibliography at least contains some references to this work. Also, I shall not attempt to trace the historical development.

The books [31], [40], [41] and [29] and the bibliographical analysis [28] give good introductions to Nevanlinna theory in general. I shall use the standard notations as given in [31]. The deficiency of the meromorphic function $f(z)$ with respect to $\infty$ is

$$
\delta(\infty, f)=\underline{\varliminf} \frac{m(r, f)}{T(r, f)}=1-\varlimsup \frac{N(r, f)}{T(r, f)}
$$

as $(r \rightarrow \infty)$. For $a \in \mathbf{C}$

$$
\delta(a, f)=\delta(\infty, 1 /(f-a))
$$

A value $c \in \hat{\mathbf{C}}$ is deficient, if $\delta(c, f)>0$. It follows from the First Main Theorem of Nevanlinna theory that

$$
0 \leqq \delta(c, f) \leqq 1 \quad(c \in \hat{\mathbf{C}})
$$


and from the Second Main Theorem that there is at most a denumerable set of $c$ with $\delta(c, f)>0$ and

$$
\sum_{c \in \hat{\mathbf{C}}} \delta(c, f) \leqq 2
$$

(Deficiency Relation or Defect Relation.) For a Picard value $a$ (=value not assumed by $f) N(a, f) \equiv 0, \delta(a, f)=1$; the defect relation implies Picard's Theorem, but asserts much more.

2. Closer analysis of the notion of deficiency. From the definition of deficient value we see that a value, $c=\infty$, say, is deficient, if and only if

$$
m(r, f)=\frac{1}{2 \pi} \int_{-\pi}^{\pi} \log ^{+}\left|f\left(r e^{i \theta}\right)\right| d \theta
$$

is of the order of magnitude of $T(r, f)$ on all large circles. This might conceivably happen, if $\log \left|f\left(r e^{i \theta}\right)\right|$ is very large compared to $T$ in a very small set on all large circles $|z|=$ const. Indeed, this can be the case, if $f(z)$ is of infinite order. But for meromorphic functions of finite order $\varrho$ or even of finite lower order

one has

$$
\lambda(=\underline{\lim } \log T(r, f) / \log r)
$$

Theorem 1 (V. P. Petrenko 1969 [43], [44]).

$$
\beta(\infty, f)=\varliminf_{r \rightarrow \infty} \frac{\log ^{+} M(r, f)}{T(r, f)} \leqq \begin{cases}\frac{\pi \lambda}{\sin \pi \lambda} & \left(0 \leqq \lambda \leqq \frac{1}{2}\right) \\ \pi \lambda & \left(\frac{1}{2}<\lambda\right) .\end{cases}
$$

This relation was conjectured by Paley in 1932, a proof for entire functions was given by N. V. Govorov shortly before Petrenko's proof (1969 [30]). Petrenko has studied the 'deviations'

$$
\beta(a, f)=\beta\left(\infty, \frac{1}{f-a}\right)
$$

in detail (summarized in [45]).

Let

$$
E(r, f)=\left\{\theta:\left|f\left(r e^{i \theta}\right)\right|>1\right\}
$$

where we think of $\theta$ as a point of the unit circle,

and

$$
|E(r, f)|=\text { Lebesgue measure of } E(r, f) \text {, }
$$

$$
\sigma(f)=\varlimsup_{r \rightarrow \infty}|E(r, f)|
$$

("The spread of $\infty$ "). 
Then

$$
\begin{aligned}
\delta(\infty, f)=\underline{\lim } m(r / f) / T(r, f) & \leqq \varliminf \lim |E(r, f)| M(r, f) / T(r, f) \\
& \leqq \sigma(f) \beta(\infty, f)
\end{aligned}
$$

and (3) implies that for a meromorphic function with lower order $\lambda<\infty$ and with $\delta(\infty, f)>0, \sigma(f)$ has a lower bound which depends on $\lambda$ and $\delta(\infty, f)$ only. The best value of this lower bound is given by

Theorem 2 (A. Baernstein 1973 [6]).

$$
\sigma(f) \geqq \min \left\{2 \pi, \frac{2}{\lambda} \arccos (1-\delta(\infty, f))\right\}=\sigma_{0}(\lambda, \delta(\infty))
$$

(This inequality is known as the "spread relation".)

Remark. There is a sequence $r_{k}$ tending to $\infty$ such that for all $a \in \hat{\mathbf{C}}$ $\sigma(1 /(f-a))=\lim _{k \rightarrow \infty}\left|E\left(r_{k}, 1 /(f-a)\right)\right|$.

The proof of Theorem 2 uses the properties of the $T^{*}$ function which was introduced by Baernstein in this connection. Its definition is as follows. Let

$$
m^{*}\left(r e^{i \varphi}\right)=\sup _{|E|=2 \varphi} \frac{1}{2 \pi} \int_{E} \log \left|f\left(r e^{i \theta}\right)\right| d \theta,
$$

where $E$ varies over all measurable sets in $(0,2 \pi)$ of Lebesgue measure $2 \varphi$. Then

$$
T^{*}\left(r e^{i \varphi}, f\right)=m^{*}\left(r e^{i \varphi}\right)+N(r, f) .
$$

This function has found many other applications, some in fields far removed from value distribution theory (see [7]). One of these is

Theorem 3 (Baernstein 1974 [8]). Let $f(z)$ be an entire function and let the real numbers $\lambda$ and $\beta$ satisfy

Then, either the set

$$
0<\lambda<\infty, \quad 0<\beta<\pi, \quad \beta \lambda<\pi
$$

$$
\left\{\theta: \log \left|f\left(r e^{i \theta}\right)\right|>\cos \beta \lambda \log M(r, f)\right\}
$$

contains an interval of length $\geqq 2 \beta$ for some arbitrarily large $r$ or

$$
\lim _{r \rightarrow \infty} r^{-\lambda} \log M(r, f)=L
$$

exists and

$$
0<L \leqq \infty
$$

Another application of the $T^{*}$ function is a result of Anderson and Baernstein (1978 [3]) which gives the exact lower bound of

$$
\sigma(\alpha, f)=\varlimsup_{r \rightarrow \infty}\left|\left\{\theta: \log \left|f\left(r e^{i \theta}\right)\right|>\alpha T(r, f)\right\}\right| \quad(\alpha>0)
$$

as $f$ runs through all meromorphic functions of lower order $\lambda$. 
Theorem 3 raises the problem of determining

$$
\sigma^{*}(f)=\varlimsup_{r \rightarrow \infty}\{\text { length of largest interval } \subset E(r, f)\}
$$

$(E(r, f)$ as in (5), $f$ of lower order $\lambda, \delta(\infty, f)>0)$.

This problem is open for $\delta(\infty, f)<1$, A. Weitsman (1977 [53]) has shown the existence of a positive lower bound for $\sigma^{*}$ depending only on $\lambda$ and $\delta(\infty, f)$; also, if $\delta(\infty, f)=1, \sigma^{*}(f) \geqq \min (2 \pi, \pi / \lambda)$, and this bound is best possible.

\section{Properties of the set $\{\delta(a, f)\}$}

(a) The inverse problem. From the early days of Nevanlinna Theory the problem of finding functions with assigned deficiencies attracted much attention. Apart from its intrinsic interest this problem was of great importance, because the attempts to solve it led to many advances and new questions in the theory of Riemann surfaces (see [29] and [41] for accounts of early work). A definitive solution to the inverse problem is given by the following theorem (we quote only the part referring to the deficiencies, Drasin also shows that the ramifications can be assigned).

Theorem 4 (Drasin 1977 [12]). Given $\left\{a_{j}\right\}_{1}^{N}\left(a_{j} \in \hat{\mathbf{C}}, a_{j}\right.$ distinct) and $\left\{\delta_{j}\right\}_{1}^{N}$, $0<\delta_{j} \leqq 1, \quad \sum_{1}^{N} \delta_{j} \leqq 2, N \leqq \infty$. Then one can find a meromorphic function $f(z)$ whose deficient values are exactly the $a_{j}$ and for all $a_{j} \delta\left(a_{j}, f\right)=\delta_{j}$. Further, if $\psi(r)$ is $a$ function tending to $\infty$ as $r \rightarrow \infty$ one can choose $f(z)$ so that

$$
\log T(r, f) / \log r<\psi(r) \quad\left(r>r_{0}\right)
$$

The proof of the theorcm uses the theory of quasi-conformal mappings in a clever way. Let us call a function $w=g(z)$ "quasi-meromorphic", if it is a quasiconformal mapping $g: \mathbf{C} \rightarrow \hat{\mathbf{C}}$, that is to say (i) the distributional partial derivatives of $g$ or $1 / g$ are locally integrable and (ii) the "complex dilatation"

$$
\mu_{g}(z)=\frac{\partial g}{\partial \bar{z}}(z) / \frac{\partial g}{\partial z}(z)
$$

satisfies

$$
\left|\mu_{g}(z)\right| \leqq k<1 \quad \text { a.e. in } \mathbf{C} \text {. }
$$

For quasi-meromorphic functions the usual definitions of $m(r, 1 /(g-a))$, $N(r, 1 /(g-a)), T(r, g)$ and $\delta(a, g)$ make sense. To prove his theorem Drasin first constructs a quasi-meromorphic $g$ with the prescribed deficiencies for which $\mu_{g}(z)$ tends to zero as $z \rightarrow \infty$ in a fairly regular fashion. He then uses the theorem that the equation

$$
\frac{\partial \psi}{\partial \bar{z}}=\mu_{g} \frac{\partial \psi}{\partial z}
$$


has a solution $w=\psi(z)$ which is a quasi-conformal homeomorphism $\psi: \mathbf{C} \rightarrow \mathbf{C}$. The function $f=g \circ \psi^{-1}$ is a quasi-conformal map $\mathbf{C} \rightarrow \hat{\mathbf{C}}$ with $\mu_{f}=0$ a.e., i.e. $f$ is meromorphic. (See [1] for the quasi-conformal background.)

Finally one deduces from the fact that $\mu_{g}(z)$ is very small for large $z$ that $f(z)$ has the right deficient values and the right deficiencies.

(b) Restrictions on the deficiencies of meromorphic functions of finite order. The function $f(z)$ in Drasin's theorem has usually infinite order. If we require that $f(z)$ is of finite order, it is no longer true that deficiencies can be assigned arbitrarily, subject only to (1) and (2). A complete set of conditions for the existence of a meromorphic function of order $\varrho>0$ with deficient values $a_{j}$ and assigned deficiencies $\delta\left(a_{j}\right)$ is not known.

One condition is given by

Theorem 5 (A. Weitsman 1972 [52]). If $f(z)$ is a meromorphic function of lower order $\mu<\infty$, then

$$
\sum_{a} \delta^{1 / 3}(a, f)<\infty .
$$

Earlier Hayman [31] had proved

$$
\sum_{a} \delta^{\alpha}(a, f)<\infty \quad\left(\alpha<\frac{1}{3}\right) .
$$

Recently G. A. Barsegjan [10] announced another proof of Theorem 4 which seems to follow the line of Hayman's proof. Barsegjan's method also yields

Theorem 6 (G. A. Barsegjan [10]). If $f(z)$ is a meromorphic function of finite lower order, then

$$
\sum_{a} \beta^{1 / 2}(a, f)<\infty
$$

where $\beta(a, f)$ is defined by (4) and (3).

The exponent $1 / 3$ in Theorem 5 is best possible. Hayman [31], refining a construction of A. A. Gol'dberg, has examples of functions of finite order with

$$
\sum \delta^{\alpha}(a, f)=\infty \quad\left(\alpha<\frac{1}{3}\right) .
$$

In particular this construction can be used to show that there are meromorphic functions of every positive order with infinitely many deficient values.

By using methods of approximation theory Arakeljan could prove

Theorem 7 (N. U. Arakeljan 1966 [5]). There are entire functions of any finite order $\varrho>1 / 2$ with infinitely many deficient values. 
The deficiencies in Arakeljan's example are very small. He conjectures that for entire functions of finite order

$$
\sum_{a}(\log (1 / \delta(a, f)))^{-1}<\infty
$$

These results make the following theorem especially noteworthy.

Theorem 8 (Weitsman 1972 [51]). If $f(z)$ is a meromorphic function of finite lower order $\lambda$ and

$$
s(f)=\sum_{a} \delta(a, f)=2,
$$

then $f(z)$ has at most $2 \lambda$ deficient values.

For other conditions which ensure that the number of deficient values is finite see $\S 4$.

(c) Functions of lower order less than one. Valiron proved in 1950 [50] that a function of order zero cannot have more than one deficient value. Edrei and Fuchs showed that this remains true for functions of lower order zero [17].

Several deficient values can occur as soon as the lower order is positive, but the values of the deficiencies are subject to restrictions.

Theorem 9 (Edrei 1969 [15]; with $\varrho$ in place of $\lambda$, Edrei and Fuchs 1960 [19]). Let $f(z)$ be a meromorphic function of lower order $\lambda(0<\lambda<1)$. Let $a, b \in \hat{\mathbf{C}}, a \neq b$, Then

$$
u=1-\delta(a, f) \quad v=1-\delta(b, f) .
$$

(i) $\sin ^{2} \pi \lambda \leqq u^{2}+v^{2}-2 u v \cos \pi \lambda$.

(ii) $v \leqq \cos \pi \lambda$ implies $u=1$.

This is an easy consequence of the "spread relation". We may assume $a=0$, $b=\infty$. With the notations (5) and (6) and using the remark after Theorem 2 we have

and so, letting $k \rightarrow \infty$

$$
\left|E\left(r_{k}, f\right)\right|+\left|E\left(r_{k}, 1 / f\right)\right| \leqq 2 \pi
$$

$$
\sigma(f)+\sigma(1 / f) \leqq 2 \pi .
$$

Replacing $\sigma$ by its bound $\sigma_{0}$ from Theorem 2 we obtain Theorem 9 .

In 1978 V. P. Petrenko and A. V. Krytov [46] proved

Theorem 10. Let $f(z)$ be meromorphic, of order $\varrho, 0<\varrho<1$. If

then

$$
\delta\left(a_{1}, f\right) \geqq \delta\left(a_{2}, f\right) \geqq \delta\left(a_{3}, f\right),
$$

$$
\delta\left(a_{3}, f\right) \leqq 1-\frac{2 \sin \pi \varrho / 2}{\pi \varrho} .
$$


The proof of Theorem 10 uses the theory of holomorphic curves. For small values of $\varrho$ this bound is better than that obtained by application of the spread relation and the inequality $\sigma\left(c /\left(f-a_{3}\right)\right) \leqq 2 \pi / 3$.

(d) Sum of deficiencies. By a refinement of the idea used in the proof of Theorem 9 it is possible to prove

Theorem 11 (Edrei 1973 [16], see also Fuchs 1969 [22]). If $f(z)$ is a meromorphic function of lower order $\lambda$, then

(i) $s(f)=\sum_{a} \delta(a, f) \leqq 1-\cos \pi \lambda \quad(0<\lambda \leqq 1 / 2)$,

unless $f(z)$ has only one deficient value, in which case the deficiency can have any value in $[0,1]$.

(ii) $s(f) \leqq 2-\sin \pi \lambda \quad(1 / 2 \leqq \lambda<1)$

and equality can hold if and only if $f(z)$ has exactly two deficient values with deficiencies

$$
\delta\left(a_{1}\right)=1, \quad \delta\left(a_{2}\right)=1-\sin \pi \lambda .
$$

A precise upper bound of $s(f)$ for functions of order $\varrho>1$ is not known. Drasin and Weitsman have given good arguments for their

Conjecture (1975 [14]). If $f(z)$ is a meromorphic function of order $\varrho$, then

$$
\begin{gathered}
s(f) \leqq \max \left\{\Lambda_{1}, \Lambda_{2}\right\}, \\
\Lambda_{1}=2-\frac{2 \sin \frac{\pi}{2}\{2 \varrho\}}{[2 \varrho]+2 \sin \frac{\pi}{2}\{2 \varrho\}}, \\
\Lambda_{2}=2-\frac{2 \cos \frac{\pi}{2}\{2 \varrho\}}{[2 \varrho]+1},
\end{gathered}
$$

where $[x]=$ largest integer $\leqq x,\{x\}=x-[x]$. Drasin and Weitsman showed that this bound cannot be replaced by a smaller one, if $f(z)$ has at least three deficient values.

For entire functions the well known inequality

$$
\sum_{a \neq \infty} \delta(a, f) \leqq \delta^{\prime}(0, f)
$$

relates the sum of deficiencies problem to the problem of the least upper bound of $\delta(0, f)+\delta(\infty, f)$ for functions $f$ of a given order. Since

$$
\begin{aligned}
\delta(0, f)+\delta(\infty, f) & =2-\varlimsup \frac{N(r, 1 / f)}{T(r)}-\varlimsup \frac{N(r, f)}{T(r)} \\
& \leqq 2-K(f),
\end{aligned}
$$


where

$$
K(f)=\varlimsup \frac{N(r, 1 / f)+N(r, f)}{T(r, f)},
$$

this problem is closely related to the problem of finding

$$
\varkappa(\varrho)=\inf K(f)
$$

as $f$ runs through all meromorphic functions of order $\varrho$.

R. Nevanlinna made a conjecture about the precise value of $\varkappa(\varrho)$ and proved that $x(\varrho)>0$, if $\varrho$ is not an integer (1929 [40]). This conjecture has been verified for entire functions of completely regular growth by A. A. Gol'dberg [27]. It is still open in the general case.

J. Miles and D. F. Shea considered a variant of the $\varkappa(\varrho)$ problem: Let

$$
\begin{gathered}
m_{2}(r, f)=\left\{\frac{1}{2 \pi} \int_{-\pi}^{\pi} \log ^{2}\left|f\left(r e^{i \theta}\right)\right| d \theta\right\}^{1 / 2}, \\
K_{2}(f)=\varlimsup_{r \rightarrow \infty}\{N(r, f)+N(r, 1 / f)\} / m_{2}(r, f) .
\end{gathered}
$$

Theorem 12 (Miles and Shea 1973 [36]). For meromorphic functions of lower order $\lambda$

$$
K_{2}(f) \geqq \frac{|\sin \pi \lambda|}{\pi \lambda}\left(\frac{1}{2}+\frac{\sin 2 \pi \lambda}{4 \pi \lambda}\right)^{-1}
$$

and this bound is best possible.

For a refinement see [37].

4. Relations between deficiencies and other properties of meromorphic functions

(a) Does the existence of deficient values have implications for the growth properties of a function?

If $f(z)$ is an entire function with a finite deficient value, then $i>1 / 2$. (By the second part of Theorem 9 with $b=\infty, \lambda \leqq 1 / 2$.) A. A. Gol'dberg [23, 24] has shown that there is an entire function $f$ with $\delta(0, f)>0$ and assigned values of $\lambda$ and $\varrho$, $1 / 2<\lambda \leqq \varrho<\infty$. But if the deficiency sum of $f(z)$ is sufficiently close to 2 , then restrictions on $\lambda$ and $\varrho$ are known.

(b) Functions with $s(f)=\sum_{a} \delta(a, f)=2$.

Theorem 13 (Edrei and Fuchs 1959 [17]). If $f(z)$ is an entire function of finite order with $s(f)=\sum \delta(a, f)=2$, then

$$
\lambda=\varrho=\text { integer } \geqq 1 .
$$

Many other properties of these functions are known. 
Theorem 14 (Pfluger 1946 [47]). Let $f(z)$ be an entire function of finite order Q. If

then (i) @ is a positive integer.

$$
s(f)=2,
$$

(ii) Each deficiency is an integral multiple of $1 / \varrho$. In particular $f(z)$ can not have more than @ finite deficient values.

Edrei and Fuchs [18] added in 1959

(iii) Each deficient value is asymptotic. [A value $c$ is asymptotic, if $f(z) \rightarrow c$ as $z \rightarrow \infty$ along a path $L$ in $\mathbf{C}$.] In the same paper they also showed that there is a constant $\varepsilon(\varrho)>0$ such that for meromorphic $f(z)$ of order $\varrho, s(f)>2-\varepsilon(\varrho), \delta(\infty, f)>$ $1-\varepsilon(\varrho)$ implies the existence of a set of at most $p$ asymptotic deficient values where $p$ is the integer closest to $\varrho$. The question whether there are any non-asymptotic deficient values under these circumstances is unsolved.

A recent extension of Theorem 14 is

Theorem 15 (Yang Lo and Chang Kuan-heo 1979 [53]). If $f(z)$ is an entire function of lower order $\lambda$ and

$$
\sum_{j=-\infty}^{\infty} \sum_{a \neq 0, \infty} \delta\left(a, f^{(j)}\right)=1
$$

then the number of deficient values of $f^{(j)}(z)$ is at most $\lambda+2$. Every finite deficient value is asymptotic and every deficiency is a multiple of $1 / \lambda$. Also

$$
\sum_{j} \sum_{a \neq 0, \infty} \delta^{1 / 3}\left(a, f^{(j)}\right)<\infty .
$$

F. Nevanlinna [39] gave examples of meromorphic functions whose order is an integral multiple of $1 / 2 \geqq 1$ for which $s(f)=2$. He made the

Conjecture. If $f(z)$ is a meromorphic function of order $\varrho$ with sum of deficiencies equal to two, then $\varrho$ is an integral multiple of $1 / 2 \geqq 1$, the number of deficient values is $\leqq 2 \varrho$ and each deficiency is a multiple of $1 / \varrho$.

Part of this conjecture is now proved:

Theorem 16 (A. Weitsman 1969 [51]). A meromorphic function of lower order

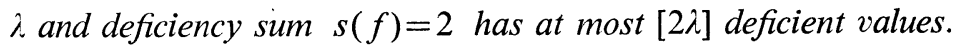

Theorem 17 (D. Drasin 1980 [13]). If $f(z)$ is a meromorphic function of order $\varrho$ with $s(f)=2$, then all deficiencies are integer multiples of $1 / k$, when $k$ is an integer $\leqq \varrho$.

(c) Functions with extremal spread. Entire functions with $s(f)=2$ give an example of a class of functions whose value distribution is very much determined by a condition on the deficiencies. 
Another example of such a class are the "functions of extremal spread", that is to say functions for which equality holds in the spread relation (7).

As a special case of a more general theorem Baernstein proved

Theorem 18 (Baernstein 1977 [9]). If $f(z)$ is a function of finite order with extremal spread, then $\lambda=\varrho$.

Examples of functions of given finite order $\varrho>0$ with extremal spread can be written down explicitly in terms of Lindelöf functions. In these examples $f$ is large in a sector and small in the complementary sector. Edrei and Fuchs [20, 21] have shown that every function of extremal spread must have a behavior very similar to the functions of these examples, at least in certain annuli $A_{n}<|z|<B_{n}, A_{n} \rightarrow \infty$, $B_{n} / A_{n} \rightarrow \infty$.

(d) Deficient values and asymptotic values. R. Nevanlinna conjectured that a deficient value of $f(z)$ must be an asymptotic value. The first counterexample was given by O. Teichmüller [49] in 1937. Theorem 7 combined with the DenjoyAhlfors-Carleman theorem proves the existence of entire functions of finite order with infinitely many non-asymptotic deficiencies. A. A. Gol'dberg [25] has shown that there are meromorphic functions of order $\varrho>1$ with non-asymptotic deficient value 0 and $\delta(0, f)=1$. He also proved [26] that there are entire functions of infinite order with zero as non-asymptotic deficient value and $\delta(0, f)=1$. (This can not happen for functions of finite order by Theorem 14 (iii).)

Conditions under which a deficient value is asymptotic are given by the following theorems. (See also [2].)

Theorem 19 (Anderson and Clunie 1966 [4]). If $f(z)$ is a meromorphic function with

$$
T(r, f)=O\left(\log ^{2} r\right)
$$

and $\delta(a, f)>0$, then a is an asymptotic value.

Hayman has constructed examples of meromorphic functions with a nonasymptotic deficient value and $T(r, f)=O\left(\psi(r) \log ^{2} r\right)$ where $\psi$ is any function increasing to $\infty$ as $r \rightarrow \infty$ [33].

Theorem 20 (Hayman 1978 [33]). The meromorphic function $f(z)$ has the asymptotic value $a$, if

$$
T(r, f)-\frac{1}{2} r^{1 / 2} \int_{r}^{\infty} N(t, a) t^{-3 / 2} d t \rightarrow \infty \quad(r \rightarrow \infty) .
$$

Corollary 1. If $T(2 r, f) / T(r, f) \rightarrow 1(r \rightarrow \infty)$, then every deficient value is asymptotic. 
Corollary 2. If $f(z)$ is of order $\varrho, 0<\varrho<1 / 2$ and if $\delta(a, f)>2 \varrho$, then $a$ is an asymptotic value.

In the opposite direction one has

Theorem 21 (Sunyer i Balaguer 1953 [48]). Let $f(z)$ be an entire function of finite order with proximate order $\varrho(r)$. If $a \neq \infty$ is an asymptotic value of $f(z)$ with asymptotic path $L$ and if

then

$$
\log |f(z)-a|<-A|z|^{o(|z|)} \quad\left(z \in L,|z|>r_{0}\right)
$$

$$
\delta(a, f)>0
$$

(e) Deficient values and angular distribution of values. The definition of $\delta(a, f)$ only takes into account the radial distribution of $a$-points of $f(z)$. It is therefore surprising that there are relations between deficiency and angular distribution of values. Here are some examples.

Theorem 22 (Hellerstein and Shea 1978 [34]). Let $f(z)$ be an entire function of finite genus $q$, whose zeros lie on $m(<\infty)$ rays arg $z=$ const.

Then one can find constants $K$ and $B(q)$ depending only on the location of the rays and (in the case of $B$ ) on $q$ such that

$$
\delta(0, f)>B(q)
$$

whenever $q>K$. As $q \rightarrow \infty, B(q) \rightarrow 1$.

Theorem 23 (Hellerstein and Shea [34]). Let $f(z)$ be a meromorphic function of finite order, with zeros $\left\{a_{n}\right\}$ and poles $\left\{b_{n}\right\}$ in the sectors

$$
\left|\arg a_{n}\right| \leqq \eta\left|\arg b_{n}-\pi\right| \leqq \eta \quad(0 \leqq \eta \leqq \pi / 6) .
$$

Denote by $q$ the least integer such that

Then

$$
\sum\left|a_{n}\right|^{-q-1}+\sum\left|b_{n}\right|^{-q-1}<\infty .
$$

where

$$
\overline{\lim } \frac{N(r, f)+N(r, 1 / f)}{T(r, f)}<A(\eta, q)
$$

$$
A(\eta, q)<\frac{C}{\log q}+D \eta \log (1 / \eta)\left(1+q \log ^{2} q\right) \quad(q>1)
$$

Since the constant $B(q)$ in Theorem 22 tends to 1 as $q \rightarrow \infty$, it is natural to conjecture that an entire function of infinite order whose zeros lie on a finite number of rays $\arg z=$ const. must have $\delta(\infty)=1$, i.e., $N(r, 1 / f) / T(r, f) \rightarrow 0$. However, one has 
Theorem 24 (J. Miles 1979 [35]). (i) Let $f(z)$ be an entire function of infinite order whose zeros lie on a finite number of rays. Then there is a set $E \subset[1, \infty)$ of logarithmic density zero such that

as $r \rightarrow \infty$ avoiding $E$.

$$
\frac{N(r, 1 / f)}{T(r, f)} \rightarrow 0
$$

(ii) Given $\chi(r) \rightarrow \infty$ as $r \rightarrow \infty$, there is an entire function of infinite order with positive zeros such that $\delta(0, f)=0$ and

$$
\frac{\log T(r, f)}{\log r}<\varkappa(r) \quad\left(r>r_{0}\right)
$$

Definition. One says that the zeros of the entire function $f(z)$ have an angular density with respect to the proximate order $\varrho(r)$, if given $\varepsilon$ one can find arguments $\varphi_{1}, \varphi_{2}, \ldots, \varphi_{k}, \varphi_{k+1}=\varphi_{1}+2 \pi$ such that $0<\varphi_{j+1}-\varphi_{j}<\varepsilon$ and

$$
\lim _{r \rightarrow \infty} n\left(r, \varphi_{j}, \varphi_{j+1}\right) r^{-\varrho(r)}
$$

exists for $j=1,2,3, \ldots, k$, where $n(r, \alpha, \beta)$ is the number of zeros of $f(z)$ in

$$
0<|z| \leqq r, \quad \alpha \leqq \arg z \leqq \beta .
$$

Theorem 25 (Oum 1969 [42]). Let $\varrho(r)$ be a proximate order of the entire function $f(z)$ of order $\varrho$. If the zeros of $f(z)$ have an angular density with respect to $\varrho(r)$ then $f(z)$ has at most $2 \varrho+1$ deficient values. If $2 \varrho$ is an integer, this bound can be replaced by $2 \varrho$.

Yang Lo and Chang Kuan-heo have investigated the relations between the number of deficient values and the number of Julia and Borel directions. A typical result is

Theorem 26 (Chang Kuan-heo 1978 [11]). Let $f(z)$ be an entire function of lower order $\lambda<\infty$. If $f(z)$ has $q$ Julia directions, $p$ finite deficient values and $s$ asymptotic values of which $s^{\prime}$ are also deficient values, then $2 p+s-s^{\prime} \leqq q$. If equality holds and $q<\infty$, then $f(z)$ has order $\lambda$ and all deficient values are asymptotic.

(f) Deficiencies and Taylor coefficients. The upper (lower) density of a sequence $\left\{c_{n}\right\}$ is defined as $\lim \sup C(n) / n(\lim \inf C(n) / n)$, where $C(n)$ is the number of non-zero $c_{k}$ with $k \leqq n$.

Theorem 27 (Edrei and Fuchs 1959 [18]). Let

$$
f(z)=\sum_{0}^{\infty} c_{n} z^{n}
$$

be an entire function of lower order $\lambda$. Let $p$ be the integer satisfying

$$
p-\frac{1}{2} \leqq \lambda<p+\frac{1}{2} \text {. }
$$


There are constants $A$ and $\varepsilon_{0}$ such that

$$
\sum \delta(a, f)>2-\frac{A \varepsilon}{(p+1)[1+\log (p+1)]} \quad\left(0<\varepsilon<\varepsilon_{0}\right)
$$

implies that the upper and lower densities $\bar{D}$ and $D$ of $\left\{c_{n}\right\}$ satisfy

$$
\frac{s}{p}(1-\varepsilon)<\underline{D} \leqq \bar{D} \leqq \frac{s}{p} .
$$

Here $s$ is an integer, $1 \leqq s \leqq p$.

Theorem 28 (Murai 1980 [38]). Let

where $n_{k+1} / n_{k} \geqq q>1$ and

$$
f(z)=\sum_{k=0}^{\infty} c_{k} z^{n_{k}} \quad(|z|<1),
$$

Then

$$
\limsup _{k \rightarrow \infty}\left|c_{k}\right|>0 \text {. }
$$

for all $a \in \mathbf{C}$.

$$
\delta(a, f)=0
$$

Theorem 29 (Hayman 1972, consequence of Theorem 3 [32]). If $f(z)$ is entire, $\lambda<\infty$ and the density of non-vanishing Taylor coefficients of $f(z)$ is zero, then $\delta(a, f)=0$ for all $a \in \mathbf{C}$.

\section{Bibliography}

[1] Ahlfors, L. V.: Lectures on quasiconformal mappings. - D. Van Nostrand Company., Inc., Princeton, N. J.-Toronto-New York-London, 1966.

[2] Anderson, J. M.: Asymptotic values of meromorphic functions of smooth growth. - Glasgow Math. J. 20, 1979, 155-162.

[31 Anderson, J. M.,and A. Baernstein: The size of the set on which a meromorphic function is large. - Proc. London Math. Soc. (3) 36, 1978, 518-539.

[4] Anderson, J. M., and J. J. Clunie: Slowly growing meromorphic functions. - Comment. Math. Helv. 40, 1966, 267-280.

[5] Arakeljan, N. U.: Entire functions of finite order with an infinite set of deficient values. Dokl. Akad. Nauk SSSR 170, 1966, 999-1002 (Russian).

[6] Baernstein, A., II: Proof of Edrei's spread conjecture. - Proc. London Math. Soc. (3) 26, 1973, 418-434.

[7] BAERnSteIN, A., II: Integral means, univalent functions and circular symmetrizations. - Acta Math. 133, 1974, 139-169.

[8] Baernstein, A., II: A generalization of the $\cos \pi \varrho$ theorem. - Trans. Amer. Math. Soc. 193, 1974, 181-197.

[9] BaERnstern, A., II: Regularity theorems associated with the spread relation. - J. Analyse Math. 31, 1977, 76-111.

[10] Barsegjan, G. A.: Deficiencies and growth of meromorphic functions of finite lower order. Akad. Nauk Armjan. SSR Dokl. 67, 1978, 291-294 (Russian). 
[11] Chang, KunN-Heo: A study of the relations between the defect values, the asymptotic values and the Julia directions of entire and meromorphic functions. - Sci. Sinica, Suppl. I, 1978, 1-80 (Chinese, English summary).

[12] Drasin, D.: The inverse problem of the Nevanlinna theory. - Acta. Math. 138, 1977, 83151.

[13] Drasin, D.: Quasiconformal modifications of functions having deficiency sum two. - To appear.

[14] Drasin, D., and A. Weitsman: Meromorphic functions with large sums of deficiencies. - Adv. in Math. 15, 1975, 93-126.

[15] EdReI, A.: Locally tauberian theorems for meromorphic functions of lower order less than one. - Trans. Amer. Math. Soc. 140, 1969, 309-332.

[16] EdReI, A.: Solution of the deficiency problem for functions of small lower order. - Proc. London Math. Soc. (3) 26, 1973, 435-445.

[17] Edrei, A., and W. H. J. Fuchs: On the growth of meromorphic functions with several deficient values. - Trans. Amer. Math. Soc. 93, 1959, 292-328.

[18] Edrei, A., and W. H. J. Fuchs: Valeurs déficientes et valeurs asymptotiques des fonctions méromorphes. - Comment. Math. Helv. 33, 1959, 258-295.

[19] EdreI, A., and W. H. J. Fuchs: The deficiencies of meromorphic functions of order less than one. - Duke Math. J. 27, 1960, 233-249.

[20] EdreI, A., and W. H. J. Fuchs: Asymptotic behavior of meromorphic functions with extremal spread I. - Ann. Acad. Sci. Fenn. Ser. A I Math. 2, 1976, 67-111.

[21] EdreI, A., and W. H. J. Fuchs: Asymptotic behavior of meromorphic functions with extremal spread II. - Ann. Acad. Sci. Fenn. Ser. A I Math. 3, 1977, 141-168.

[22] Fuchs, W. H. J.: Meromorphic functions of lower order less than one. - Mat. Science Report 64, Madras, 1969.

[23] Gol'Dberg, A. A.: On the possible value of the lower order of an entire function with a finite deficient value. - Dokl. Akad. Nauk SSSR 159, 1964, 968-970 (Russian).

[24] Gol'Dberg, A. A.: On the lower order of an entire function with a finite defective value. Sibirsk, Mat. Ž. 5, 1964, 54-76 (Russian).

[25] Gol'dberg, A. A.: Non-asymptotic defect values of meromorphic functions. - Dokl. Akad. Nauk SSSR 171, 1966, 254-257 (Russian).

[26] Gol'dberg, A. A.: Non-asymptotic defect values of entire functions of infinite order. - Mat. Zametki 1, 1967, 81-86 (Russian).

[27] Gol'dberg, A. A.: The defects of entire functions with completely regular growth. - Teor. Funkciĭ Funkcional. Anal. i Priložen, 14, 1971, 88-101 (Russian).

[28] Gol'Dberg, A. A.: Meromorphic functions. - Mathematical analysis Vol. 10, Akad. Nauk SSSRVsesojuz. Inst. Naučn. Tehn. Informacii, Moscow, 1973, 5-97 (Russian).

[29] Gol'Dberg, A. A., and I. V. Ostrovskiř: The distribution of values of meromorphic functions. - Izdat. "Nauka", Moscow, 1970 (Russian).

[30] Govorov, N. V.: The Paley conjecture. - Funkcional. Anal. i Priložen. 3, 1969, 41-45.

[31] Hayman, W. K.: Meromorphic functions. - Clarendon Press, Oxford, 1964.

[32] Hayman, W. K.: Angular value distribution of power series with gaps. - Proc. London Math. Soc. (3) 24, 1972, 590-624.

[33] Hayman, W. K.: On Iversen's theorem for meromorphic functions with few poles. - Acta Math. 141, 1978, 115-145.

[34] Hellerstein, S., and D. F. Shea: Minimal deficiencies for entire functions with radially distributed zeros. - Proc. London Math. Soc. (3) 37, 1978, 35-55.

[35] Miles, J.: On entire functions of infinite order with radially distributed zeros. - Pacific J. Math. $81,1979,131-157$. 
[36] Miles, J., and D. F. SheA: An extremal problem in value distribution theory. - Quart. J. Math. Oxford Ser. (2) 24, 1973, 377-383.

[37] Miles, J., and D. F. SHEA: On the growth of meromorphic functions having at least one deficient value. - Duke Math. J. 43, 1976, 171-186.

[38] MuraI, T.: Sur la distribution des valeurs des séries lacunaires. - J. London Math. Soc. (2) (to appear).

[39] Nevanlinna, F.: Über eine Klasse meromorpher Funktionen. - C.R. Septième Congrès Math. Scand., Oslo, 1930, 81-83.

[40] Nevanlinna, R.: Le théorème de Picard-Borel et la théorie des fonctions méromorphes. Gauthier-Villars et $C^{\text {ie }}$, Paris, 1929.

[41] Nevanlinna, R.: Eindeutige analytische Funktionen. 2nd edition. - Springer-Verlag, BerlinGöttingen-Heidelberg, 1953.

[42] Oum, KI-CHoul: Bounds for the numbers of deficient values of entire functions whose zeros have angular densities. - Pacific J. Math. 29, 1969, 187-202.

[43] Petrenko, V. P.: Growth of meromorphic functions of finite lower order. - Dokl. Akad. Nauk SSSR 184, 1969, 1031-1033 (Russian).

[44] Petrenko, V. P.: Growth of meromorphic functions of finite lower order. - Izv. Akad. Nauk SSSR Ser. Mat. 33, 1969, 414-454 (Russian).

[45] Petrenko, V. P.: Growth of meromorphic functions. - Izdat. pri Hark'ov. Gos. Univ. Izdat. Ob'ed. "Višča Škola", Kharkov, 1978 (Russian).

[46] Petrenko, V. P., and A. V. Krytov: A remark on the defects of meromorphic functions. Questions of mathematical physics and functional analysis, (Proc. Res. Sem. Inst. Low Temp. Phys. Engrg., Kharkov) "Naukova Dumka”, Kiev, 1976, 33—42 (Russian).

[47] Pfluger, A.: Zur Defektrelation ganzer Funktionen endlicher Ordnung. - Comment. Math. Helv. 19, 1946, 91-104.

[48] Sunyer i Balanguer, F.: Sur le théorème de Denjoy-Carleman-Ahlfors. - C. R. Acad. Sci. Paris 237, 1953, 548-550.

[49] Teichmüller, O.: Eine Umkehrung des zweiten Hauptsatzes der Werteverteilungslehre. Deutsche Math. 2, 1937, 96-107.

[50] Valiron, G.: Sur les valeurs déficientes des fonctions méromorphes d'ordre nul. - C. R. Acad. Sci. Paris 230, 1950, 40-42.

[51] Weitsman, A.: Meromorphic functions with maximal deficiency sum and a conjecture of F. Nevanlinna. - Acta Math. 123, 1969, 115-139.

[52] Weitsman, A.: A theorem on Nevanlinna deficiencies. - Acta Math. 128, 1972, 41-52.

[53] Weitsman, A.: Some remarks on the spread of a Nevanlinna deficiency. - Institut MittagLeffler Report No. 7, 1977.

[54] YANG, Lo., and Chang KuAN-Heo: Deficient values and asymptotic values of entire functions. - Sci. Sinica, Suppl. II, 1979, 190-203 (Chinese).

\section{Bibliography on holomorphic curves}

[55] Ahlfors, L.V.: The theory of meromorphic curves. - Acta Soc. Sci. Fennicae. Nova Ser. A $3: 4,1941,1-31$.

[56] Cartan, H.: Sur les zéros des combinaisons linéaires de $p$ fonctions holomorphes données. Mathematica, Cluj 7, 1933, 1-31.

[57] Cowen, M., and P. A. Griffiths: Holomorphic curves and metrics of negative curvature. - J. Analyse Math. 29, 1976, 93-153.

[58] Green, M. L.: Holomorphic maps into complex projective space omitting hyperplanes. - Trans. Amer. Math. Soc. 169, 1972, 83-103. 
[59] Griffiths, P. A.: Entire holomorphic mappings in one and several complex variables. - Annals of Mathematics Studies 85, Princeton University Press, and the University of Tokyo Press, Tokyo, 1976.

[60] Krutīn', V. İ.: The values of positive deviations and values of defects of entire curves of finite lower order. - Izv. Akad. Nauk SSSR Ser. Mat. 42, 1978, 1021-1049 (Russian).

[61] Krytov, A. V.: Defects of entire curves of finite lower order. - Ukrain. Mat. Ž. 31, 1979, 273-278 (Russian).

[62] MoRI, S.: Holomorphic curves with max deficiency sum. - Kōdai Math. J. 2, 1979, 116122.

[63] Ninō, K.: Deficiencies of the associated curves of a holomorphic curve in the projective space. - Proc. Amer. Math. Soc. 59, 1976, 81-88.

[64] NıINō, K.: Spread relation and value distribution in an angular domain of holomorphic curves. - Kōdai Math. Sem. Rep. 28, 1976/77, 361-371.

[65] Noguchi, J.: Holomorphic curves in algebraic varieties. - Hiroshima Math. J. 7, 1977, 833-853.

[66] Petrenko, V. P.: The growth of entire curves of lower order $\lambda<1$. - Teor. Funkciĭ Funkcional. Anal. i Priložen. 20, 1974, 134-141 (Russian).

[67] Petrenko, V. P.: On a regularity in the growth of meromorphic functions and of entire curves. Ukrain. Mat. Ž. 30, 1978, 627-633 (Russian).

[68] Petrenko, V. P., and M. Hussaľn: The growth of entire curves. - Izv. Akad. Nauk SSSR Ser. Mat. 37, 1973, 466-477 (Russian).

[69] Petrenko, V. P., and M. HussaǏn: The defects of entire curves of lower order $\lambda<1$. Teor. Funkciǐ Funkcional. Anal. i Priložen. 24, 1975, 128-138 (Russian).

[70] Shiffman, B.: Holomorphic curves in algebraic manifolds. - Bull. Amer. Math. Soc. 83, 1977, $553-568$.

[71] Wu, H.: The equidistribution theory of holomorphic curves. - Annals of Mathematics Studies 64, Princeton University Press and the University of Tokyo Press, Princeton, N. J., 1970.

Cornell University

Department of Mathematics

Ithaca, New York 14853

USA

Received 5 March 1981 